\title{
Early versus standard initiation of renal replacement therapy in furosemide stress test non-responsive acute kidney injury patients (the FST trial)
}

Nuttha Lumlertgul 1,2, Sadudee Peerapornratana ${ }^{1,2}$, Thananda Trakarnvanich ${ }^{3}$, Wanjak Pongsittisak ${ }^{3}$, Kajbundit Surasit ${ }^{4}$, Anan Chuasuwan ${ }^{5}$, Pleumjit Tankee ${ }^{6}$, Khajohn Tiranathanagul', Kearkiat Praditpornsilpa', Kriang Tungsanga ${ }^{1}$, Somchai Eiam-Ong ${ }^{1}$, John A. Kellum ${ }^{7}$, Nattachai Srisawat ${ }^{1,2,7^{*}}$ and for the FST Study Group

\begin{abstract}
Background: The timing of initiation of renal replacement therapy (RRT) in severe acute kidney injury (AKI) remains controversial, with early initiation resulting in unnecessary therapy for some patients while expectant therapy may delay RRT for other patients. The furosemide stress test (FST) has been shown to predict the need for RRT and therefore could be used to exclude low-risk patients from enrollment in trials of RRT timing. We conducted this multicenter pilot study to determine whether FST could be used to screen patients at high risk for RRT and to determine the feasibility of incorporating FST into a trial of early initiation of RRT.

Methods: FST was performed using intravenous furosemide $(1 \mathrm{mg} / \mathrm{kg}$ in furosemide-naive patients or $1.5 \mathrm{mg} / \mathrm{kg}$ in previous furosemide users). FST-nonresponsive patients (urine output less than $200 \mathrm{~mL}$ in $2 \mathrm{~h}$ ) were then randomized to early (initiation within $6 \mathrm{~h}$ ) or standard (initiation by urgent indication) RRT.

Results: FST was completed in all patients (100\%). Only 6/44 (13.6\%) FST-responsive patients ultimately received RRT while 47/60 (78.3\%) nonresponders randomized to standard RRT either received RRT or died $(P<0.001)$. Among 118 FST-nonresponsive patients, 98.3\% in the early RRT arm and 75\% in the standard RRT arm received RRT. The adherence to the protocol was $94.8 \%$ and $100 \%$ in the early and standard RRT group, respectively. We observed no differences in 28-day mortality (62.1 versus 58.3\%, $P=0.68)$, 7-day fluid balance, or RRT dependence at day 28 . However, hypophosphatemia occurred more frequently in the early RRT arm $(P=0.002)$.
\end{abstract}

Conclusion: The furosemide stress test appears to be feasible and effective in identifying patients for randomization to different RRT initiation times. Our findings should guide implementation of large-scale randomized controlled trials for the timing of RRT initiation.

Trial registration: clinicaltrials.gov, NCT02730117. Registered 6 April 2016.

Keywords: Furosemide stress test, Acute kidney injury, Renal replacement therapy

\footnotetext{
* Correspondence: drnattachai@yahoo.com

'Division of Nephrology, Department of Medicine, Faculty of Medicine,

Chulalongkorn University, Bangkok, Thailand

${ }^{2}$ Excellence center for Critical Care Nephrology, King Chulalongkorn

Memorial Hospital, Thai Red Cross Society, Bangkok, Thailand

Full list of author information is available at the end of the article
} 


\section{Background}

Acute kidney injury (AKI) is one of the most common and serious complications in critical care patients [1-3]. Renal replacement therapy (RRT) provides cornerstone management in severe AKI. While RRT is initiated promptly for life-threatening indications (e.g., severe hyperkalemia), there is controversy as to whether earlier initiation is beneficial in the absence of urgent indications [4-6]. Two recent randomized trials reached opposite conclusions as to whether early initiation is beneficial [7, 8]. The Artificial Kidney Initiation in Kidney Injury (AKIKI) trial used AKI stage 3 and conventional indications for RRT initiation. Early initiation was not superior to a conservative approach, and the lowest mortality was observed in patients who never received RRT. In the standard RRT group, $49 \%$ of the patients spontaneously recovered [8]. In the Effect of Early vs Delayed Initiation of Renal Replacement Therapy on Mortality in Critically Ill Patients with Acute Kidney Injury (ELAIN) study, however, AKI stage 2 plus plasma neutrophil gelatinase-associated lipocalin (NGAL) more than $150 \mathrm{ng} / \mathrm{mL}$ were used as inclusion criteria. Patients in the standard group had a 90\% chance of RRT. This shows that using a biomarker could be an important prognostic criterion for the prediction of RRT requirement.

Unfortunately, it is difficult to predict the requirement for RRT, and clinicians vary widely in their decision making regarding when to initiate therapy. Recently, the furosemide stress test (FST) has been validated in patients with AKI stage 1 and 2 by the Kidney Disease Improving Global Outcomes (KDIGO) criteria as a novel test for the prediction of progression to AKI stage 3, the need for RRT, and in-hospital death [9]. FST also outperformed several novel biomarkers for the prediction of adverse outcomes [10]. Therefore, FST might be suitable to risk stratify AKI patients in guiding the decision to initiate RRT.

However, before embarking on a large trial to test alternate strategies for RRT initiation, we sought to determine whether FST could be used in a clinical trial setting to stratify AKI patients and to determine the feasibility of using FST in this setting. We therefore conducted a pilot study comparing early or standard initiation of RRT in FST-nonresponsive AKI patients. We also examined 28day mortality and other clinical outcomes, although we did not power our study for these endpoints.

\section{Methods}

\section{Trial design and oversight}

The FST trial was funded by the Kidney Foundation of Thailand. The study was a prospective, multicenter, openlabel, two-group randomized trial conducted in five intensive care units (ICU) in Thailand from March 2016 to July 2017. The trial was registered at clinicaltrials.gov (NCT02730117). The institutional ethics boards of all participating centers approved the protocol. The investigators informed patients or their surrogates about the trial both orally and with a written document. Informed consent was obtained from participating patients or their substitute decision-makers before the FST was performed. Coinvestigators at each participating site were responsible for enrolling patients, ensuring adherence to the protocol, and completing the case record form. All analyses were performed by an independent statistician in accordance with the International Conference on Harmonization Good Clinical Practice Guidelines.

\section{Patients}

All adult patients ( $\geq 18$ years old) admitted to the ICU were screened. Patients with AKI at any stage (defined by KDIGO criteria) [11] were assessed for additional inclusion criteria (both of the following needed to be fulfilled): 1) clinical diagnosis of acute tubular necrosis (e.g., the presence of granular or epithelial cast, fractional excretion of sodium $\geq 1 \%$, fractional excretion of urea $\geq 50 \%$, plasma NGAL $\geq 150 \mathrm{ng} / \mathrm{mL}$ ); 2 ) in the opinion of the treating team, the patient was well resuscitated and euvolemic; and 3) in the opinion of the treating team, the patient had neither an emergent indication nor a contraindication to RRT (Additional file 1: Appendix 1). We excluded patients with any of the following criteria: 1) baseline serum creatinine $\geq 2 \mathrm{mg} / \mathrm{dL}$ (male) or $\geq 1.5 \mathrm{mg} / \mathrm{dL}$ (female) [12]; 2) history of renal allograft; 3 ) known pregnancy; 4) allergy or known sensitivity to loop diuretics; 5) moribund patients with expected death within $24 \mathrm{~h}$ or whose survival to 28 days was unlikely due to an uncontrollable comorbidity (i.e., end-stage liver or heart disease, untreatable malignancy); 6) patients with advanced directives who issued the desire not to be resuscitated; 7) prior treatment with RRT within 30 days; 8) serum albumin <2 $\mathrm{g} / \mathrm{dL}$; and 9) patients receiving extracorporeal membrane oxygenation or circulatory assistance (Additional file 1: Appendix 1). We considered the patients to be provisionally eligible if all the inclusion criteria were met, and no exclusion criteria were present.

\section{Furosemide stress test (FST)}

FST was performed by giving intravenous furosemide $1 \mathrm{mg} / \mathrm{kg}$ to naive patients or $1.5 \mathrm{mg} / \mathrm{kg}$ to patients with a history of furosemide use within 7 days. Urine output was measured hourly and, if the urine output exceeded $200 \mathrm{~mL}$ for the subsequent $2 \mathrm{~h}$, the patient was considered to be FST responsive. Patients with a urine output less than $200 \mathrm{~mL}$ in $2 \mathrm{~h}$ were considered FST nonresponsive and underwent randomization. Additional data are provided in Additional file 1: Table S1 [9].

\section{Randomization}

We randomized patients $1: 1$ to early or standard RRT initiation using a randomly permuted block of four, 
stratified by center and type of ICU. Patients randomized to early RRT were to receive RRT within $6 \mathrm{~h}$ of randomization [7]. The 6-h period was for the establishment of vascular access and RRT initiation. In the standard RRT group, RRT was initiated only if one of the following criteria were met: blood urea nitrogen $\geq 100 \mathrm{mg} / \mathrm{dL}$, serum potassium $>6 \mathrm{mmol} / \mathrm{L}$, serum bicarbonate $<12 \mathrm{mmol} / \mathrm{L}$ or $\mathrm{pH}<7.15, \mathrm{PaO}_{2} / \mathrm{FiO}_{2}$ ratio $<200$, or chest radiograph compatible with pulmonary edema.

\section{Interventions}

The starting RRT modality was continuous venovenous hemofiltration $(\mathrm{CVVH})$ using integrated machines (The Prismaflex $^{\oplus}$ system, Gambro, Sweden or the Aquarius ${ }^{\mathrm{ms}}$ system, Nikkiso, Japan) with high-flux hemofilters (AN69 or HF12) and pre-filter replacement fluid of 25$30 \mathrm{~mL} / \mathrm{kg} / \mathrm{h}$. The blood flow target was $150-200 \mathrm{~mL} / \mathrm{min}$. Regional citrate anticoagulant was the first-line of the anticoagulation strategy, followed by heparin in patients requiring systemic anticoagulation, and no anticoagulation in patients with contraindications to citrate or coagulopathy. Once the patients became hemodynamically stable (a decreasing dose or no longer needing the use of inotropic drugs) or were transferred out of the ICU, the RRT modality could be switched to prolonged intermittent RRT (PIRRT) or intermittent hemodialysis according to the judgment of the treating physician. RRT was continued until death, patient withdrawal, or renal recovery. The anticoagulant of choice in our study was intravenous heparin. However, if the patients had a contraindication to heparin, we used a no anticoagulant strategy. Renal recovery was defined as spontaneous diuresis more than $1000 \mathrm{~mL} /$ day or $2000 \mathrm{~mL} /$ day with diuretics with resolution of electrolyte or acid-base abnormalities and did no requirement to resume RRT for at least 7 days [8].

\section{Follow-up and data collection}

We followed all patients for 28 days from randomization or until hospital discharge and serially assessed the severity of illness, laboratory data, and physiological data. RRT use and prescription details were recorded. We collected blood samples at days 0,3 , and 7 to measure: serum $\mathrm{N}$-terminal prohormone of brain natriuretic peptide (NT-proBNP), a marker of volume overload [13]; plasma NGAL, a marker of kidney injury [14]; and serum angiopoietin-2, a marker of endothelial dysfunction [15].

\section{Outcomes}

The primary outcome was feasibility as judged by: 1) compliance with the study protocol for $>90 \%$ of patients; 2) the ability to use FST to differentiate the RRT rate in FST responders and standard group of nonresponders $50 \%$; 3) successful randomization of FST nonresponders; 4) separation of timing of RRT initiation between the early and standard RRT groups for at least $24 \mathrm{~h}$; and 5) $<10 \%$ lost to follow-up.

Secondary outcomes included: 1) 28-day all-cause mortality; 2) 7-day fluid balance; 3) ICU-free days; 4) mechanical ventilator-free days; 5) RRT-free days; 6) length of ICU stay and hospital stay; 7) renal recovery; 8) dialysis requirement on day 28 ; 9) the proportion of patients free from RRT on days 0,3 , and 7 ; 10) nonrenal Sequential Organ Failure Assessment (SOFA) score on days 0,3 , and 7; and 11) RRT-related adverse events and vascular access-related adverse events (Additional file 1: Appendix 2). Exploratory endpoints included biomarkers (plasma NGAL and angiopoietin-2, and serum NTproBNP) on days 0,3 , and 7 .

\section{Biomarker assays}

Blood samples were drawn in pyrogen-free vials and plasma was separated by centrifugation and frozen $\left(-80{ }^{\circ} \mathrm{C}\right)$. Blood samples for NGAL, angiopoietin-2, and NT-proBNP were determined at trial inclusion, and day 3 and day 7 after randomization. Plasma biomarkers (NGAL and angiopoietin-2) were tested using enzymelinked immunosorbent assay (R\&D Systems, USA). Serum NT-proBNP was determined using electrochemiluminescence immunoassay analysis (Cobas assay; Roche Diagnostics, Mannheim, Germany).

\section{Sample size determination}

To detect a $10.0 \%$ difference in 28 -day mortality rate between early and standard RRT with a power of $80 \%$ and a $5 \%$ significance level on the basis of a previous report, approximately 900 patients would be needed [7]. As a feasibility study, we aim to use FST to risk stratify patients who would need RRT and not need RRT. At least thirty patients were required to detect a $50 \%$ absolute difference in the proportion of RRT between FST responders and FST nonresponders (standard group) with a power of $80 \%$ $(\beta=0.2)$ at a $5 \%$ significance level $(\alpha=0.05)$. The trial was terminated on 31 July 2017 after recruiting 162 patients (44 FST responders and 118 FST nonresponders).

\section{Statistical analysis}

All analyses adhered to the intention-to-treat principle. Categorical data are described as numbers and percentages and compared between treatment groups using Chi-square or Fisher's exact test. Continuous variables are described as means (with standard deviations (SD)) or medians (with interquartile range (IQR)) and compared between each group using unpaired $t$ test in normally distributed data or Wilcoxon rank sum test for non-normal data. Overall survival for all patients was estimated by the Kaplan-Meier method. A log-rank test was used to compare time to death between treatment arms and secondarily among patients undergoing RRT 
versus no RRT, and for patients with positive versus negative FST. The univariate Cox proportional hazard regression model was used to determine factors associated with RRT requirement in the standard arms using $p$ values $<0.10$, and a multivariate model was analyzed using significant factors from the univariate model, including gender. Data from all the patients were censored at the time of death or at day 28. Severity score, laboratory data, and physiological data between days 0,3 , and 7 were computed using repeated measures analysis of variance (ANOVA) for differences within groups and generalized estimating equations for differences between groups. All analyses were performed using Stata 14.0.

\section{Results}

\section{Cohort characteristics and feasibility outcomes}

Among 297 patients with AKI potentially eligible for inclusion in this trial, 162 patients underwent FST (Fig. 1). Forty-four patients were FST responsive, while 118 patients were FST nonresponsive and were randomized to early RRT $(n=58)$ or standard RRT $(n=60)$. Compliance with the study protocol for all patients is shown in Table 1. Sites were able to perform FST in all eligible patients. The FST successfully excluded patients at low risk for RRT: 6/ 44 (13.6\%) of FST-responsive patients subsequently underwent RRT. Conversely, among FST-nonresponsive patients randomized to standard RRT, 45/60 (75\%) underwent RRT $(P<0.001)$. A comparison of baseline characteristics between FST-responsive and -nonresponsive patients is provided in Additional file 1: Table S1. Significantly fewer FST-responsive patients were on vasopressors $(P=0.016)$, had lower severity scores (Acute Physiology and Chronic Health Evaluation (APACHE) II and SOFA score; $P<0.001)$, and less severe AKI $(P=0.001)$ compared with FST-nonresponsive patients. Sepsis was present in 52.3\% of FST-responsive patients compared with $58.5 \%$ of FST-nonresponsive patients. Of the 44 FST-responsive patients, $34.1 \%$ died, whereas $60.2 \%$ of the FST-nonresponsive patients died $(P=0.003)$. When visualized by receiver operating characteristics, FST had a higher area under the curve (AUC) (0.83) than APACHE II (0.71), SOFA (0.75), and nonrenal SOFA score $(0.72)$ for the prediction of RRT (Additional file 1: Table S2).

Randomization appeared to be successful since baseline characteristics were well balanced between treatment arms, except for APACHE II score (Table 2). The early RRT group had a significantly higher APACHE II score compared with the standard RRT group (24.5 versus $21.8, P=0.027)$. Most patients had AKI stage 2 and 3 (80.0\%). Sepsis was present in 58.6\% (Table 2).

Median time from randomization to RRT initiation was 2 (IQR 1-3) $\mathrm{h}$ in the early RRT group and 21 (IQR 17-49) $\mathrm{h}$ in the standard RRT group (difference $=19 \mathrm{~h} ; P<0.001$ ). The median time from ICU admission to RRT initiation and median time from oliguria to RRT initiation in the early and standard RRT groups was 22 versus $100 \mathrm{~h}$ and

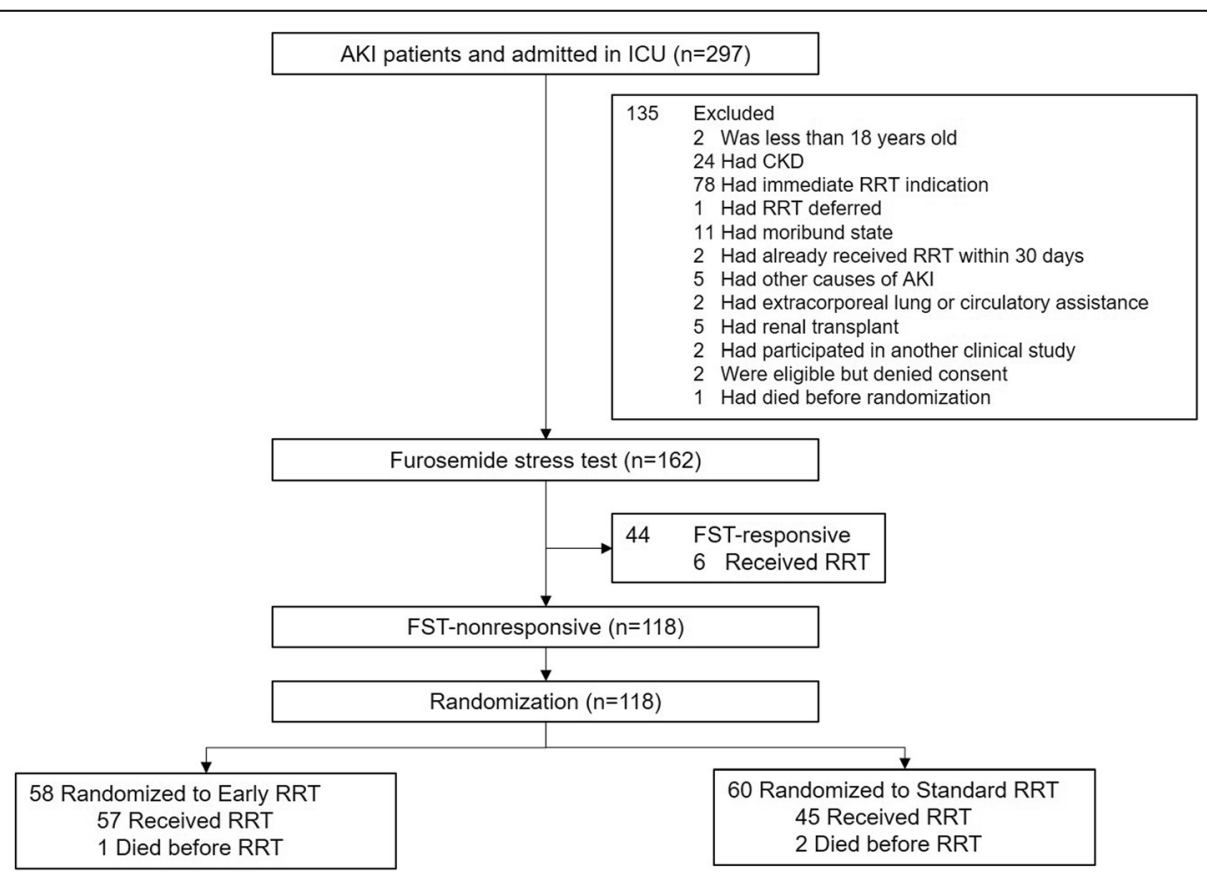

Fig. 1 Flowchart of patient allocation. AKI, acute kidney injury; CKD, chronic kidney disease; FST, furosemide stress test; ICU, intensive care unit; RRT renal replacement therapy 
Table 1 Study protocol compliance

\begin{tabular}{|c|c|c|c|}
\hline \multirow[t]{2}{*}{ Parameters } & \multicolumn{2}{|c|}{ FST nonresponsive $(n=118)$} & \multirow{2}{*}{$\begin{array}{l}\text { FST responsive } \\
(n=44)\end{array}$} \\
\hline & Early RRT $(n=58)$ & Standard RRT $(n=60)$ & \\
\hline FST completion, $n(\%)$ & $58(100)$ & $60(100)$ & $44(100)$ \\
\hline RRT, $n(\%)$ & $57(98.3)$ & $45(75)$ & $6(13.6)$ \\
\hline Initiation of RRT within $6 \mathrm{~h}$ of randomization, $n(\%)$ & $49 / 58(84.5 \%)^{\mathrm{a}}$ & N/A & N/A \\
\hline Initiation of RRT within $12 \mathrm{~h}$ of randomization, $n(\%)^{\mathrm{b}}$ & $55 / 58(94.8)$ & N/A & N/A \\
\hline Adherence to standard RRT initiation, $n(\%)$ & N/A & $45 / 45(100)$ & $6 / 6(100)$ \\
\hline Death after meeting RRT criteria but prior to RRT initiation, $n$ (\%) & $1(1.7)$ & $2(3.3)$ & $0(0)$ \\
\hline Loss to follow-up, $n(\%)$ & $0(0)$ & $0(0)$ & $0(0)$ \\
\hline
\end{tabular}

FST, furosemide stress test; N/A, not applicable; RRT, renal replacement therapy

${ }^{\mathrm{a}}$ Early RRT $=$ RRT initiation within $6 \mathrm{~h}$ after randomization; standard RRT $=$ RRT initiation according to standard indications

${ }^{\mathrm{b}}$ One patient died before RRT initiation. Two patients received RRT but later than $12 \mathrm{~h}$ due to the necessity for intervention

17 versus $38 \mathrm{~h}(P<0.001)$ in both groups (Table 3$)$. No patients were lost to follow-up for the survival status at day 28.

In the early RRT arm, 57 out of 58 patients received RRT as 1 patient died before RRT initiation. In the standard RRT group, 45 out of 60 (75.0\%) eventually met the prespecified indications and received RRT and 2 died prior to RRT. Interestingly, 15 out of 60 (25\%) showed spontaneous renal recovery (Fig. 1). In the standard arm, multivariate Cox proportional hazard regression analysis showed that SOFA score, sepsis, and baseline plasma NGAL were significant predictors for RRT requirement. Patients who spontaneously recovered had median baseline plasma NGAL level of 518.5 (IQR 397.5-641.5) ng/mL compared with 885.5 (IQR 450-1320) ng/mL in those who eventually required RRT. Plasma NGAL had an adjusted hazard ratio (HR) of 1.06 (95\% confidence interval (CI) 1. 01-1.12; $P=0.024)$ for RRT requirement.

Cumulative fluid balance from ICU admission to randomization was comparable between both groups (4763 (IQR 2837-8515) $\mathrm{mL}$ in the early group versus 5114 (IQR 2050-8803) $\mathrm{mL}$ in the standard group). RRT prescription including CVVH dose and median ultrafiltration rate per day did not differ between both groups.

\section{Secondary outcomes}

Mortality rates were estimated by the Kaplan-Meier method. The overall mortality at day 28 was $60.2 \%$. The 28 -day mortality rate in the early RRT group did not differ from the standard RRT group (62.1\% versus $58.3 \%$, $P=0.68$; unadjusted HR 0.96 (95\% CI 0.60-1.53), $P=0$. 87) (Fig. 2). Adjusted HR for APACHE II was 1.06 (95\% CI $0.66-1.69 ; P=0.81)$. The mortality rate between RRT and no RRT in the standard RRT group was also not different (HR for RRT versus no RRT 1.59 (95\% CI 0.85-4. 97), $P=0.11$ ) (Additional file 1: Figure $S 1$ ).

There were no significant differences in renal recovery, cumulative fluid balance on the first 7 days, RRT-free days, mechanical ventilation-free days, ICU-free days, or dialysis dependence on day 28 between the two groups (Table 4). The levels of plasma NGAL, NT-proBNP, and angiopoietin-2 at the time of randomization were high. There were no significant differences in these three biomarkers on days 0,3 , and 7 within treatment arm and between treatment arms (Additional file 1: Table S3).

\section{Adverse effects}

RRT-related and central venous catheter (CVC)-related adverse events are shown in Additional file 1: Table S4. There was significantly more hypophosphatemia in the early RRT group $(P=0.002)$. There were more CVCrelated malfunctions and an incidence of air embolism in the early RRT group $(P=0.038)$. Other RRT-related and CVC-related adverse events were comparable (Additional file 1: Table S4).

\section{Discussion}

In this pilot randomized controlled trial (RCT), we demonstrated the feasibility and safety of conducting a trial comparing early versus standard RRT using FST as an initial triage strategy. The results of the present study demonstrate that FST was easy to administer in the context of a clinical trial (100\% compliance) and provided excellent predictive ability for the subsequent use of RRT; nonresponsive patients had an RRT rate of 75\% versus 13 . $6 \%$ for FST-responsive patients (Additional file 1: Table S1). Compliance with other aspects of the study protocol was also excellent, with $>95 \%$ of patients receiving the intervention they were randomized to receive. Randomization was successful in that few baseline differences were seen between intervention arms and the separation of timing of initiation (early versus standard) approached, but did not quite achieve, $24 \mathrm{~h}$. Finally, we achieved excellent followup, with $100 \%$ of patients available for survival analysis.

We did not encounter any safety issues using the FST, and the only adverse events encountered with early initiation were increased rates of hypophosphatemia and dialysis catheter issues (Additional file 1: Table S4). Our 
Table 2 Demographic, clinical, and biochemical data between early RRT and standard RRT patients

\begin{tabular}{|c|c|c|c|}
\hline Parameters & $\begin{array}{l}\text { Early RRT } \\
(n=58)\end{array}$ & $\begin{array}{l}\text { Standard RRT } \\
(n=60)\end{array}$ & $P$ value \\
\hline Age (years), mean (SD) & $67.5(15.0)$ & $66.7(16.7)$ & 0.80 \\
\hline Male, $n(\%)$ & $29(50)$ & $29(48.3)$ & 0.86 \\
\hline ICU, $n(\%)$ & & & 0.79 \\
\hline Medical & $40(69)$ & $40(66.7)$ & \\
\hline Surgical & $18(31)$ & $20(33.3)$ & \\
\hline Mechanical ventilation, $n$ (\%) & $48(82.8)$ & $50(83.3)$ & 0.93 \\
\hline Vasopressors, $n(\%)$ & $45(77.6)$ & $47(78.3)$ & 0.92 \\
\hline Sepsis, $n(\%)$ & $37(63.8)$ & $32(53.3)$ & 0.25 \\
\hline APACHE II score, mean (SD) & $24.5(6.4)$ & $21.8(6.9)$ & 0.027 \\
\hline SOFA score, mean (SD) & $12.7(3.3)$ & $11.4(4.0)$ & 0.058 \\
\hline Nonrenal SOFA score, mean (SD) & $9.9(3.3)$ & $9.1(4.1)$ & 0.21 \\
\hline Baseline serum creatinine (mg/dL), mean (SD) & $1.14(0.44)$ & $1.03(0.37)$ & 0.16 \\
\hline Estimated GFR $\left(\mathrm{mL} / \mathrm{min} / 1.73 \mathrm{~m}^{2}\right)$, mean $(\mathrm{SD})^{\mathrm{a}}$ & $70.31(28.1)$ & $69.98(22.8)$ & 0.95 \\
\hline AKI staging, $n(\%)$ & & & 0.06 \\
\hline 1 & $11(19)$ & $12(20)$ & \\
\hline 2 & $27(46.6)$ & $16(26.7)$ & \\
\hline 3 & $20(34.5)$ & $32(53.3)$ & \\
\hline Blood urea nitrogen at enrollment (mg/dL), median (IQR) & $42(37-78)$ & $51(37.5-61.25)$ & 0.52 \\
\hline Serum creatinine at enrollment (mg/dL), median (IQR) & $2(2-3)$ & $2.5(2-3)$ & 0.88 \\
\hline \multicolumn{4}{|l|}{ Co-morbidities, $n$ (\%) } \\
\hline Hypertension & $29(50)$ & $24(56.7)$ & 0.47 \\
\hline Diabetes & $14(24.1)$ & $15(25)$ & 0.91 \\
\hline Dyslipidemia & $16(27.6)$ & $16(26.7)$ & 0.91 \\
\hline Ischemic heart disease & $12(20.7)$ & $10(16.7)$ & 0.58 \\
\hline Malignancy & $12(20.7)$ & $8(13.3)$ & 0.29 \\
\hline Cerebrovascular disease & $5(8.6)$ & $7(11.7)$ & 0.58 \\
\hline Chronic liver disease & $10(17.2)$ & $11(18.3)$ & 0.88 \\
\hline \multicolumn{4}{|l|}{ Nephrotoxic drugs, n (\%) } \\
\hline Colistin & $5(8.6)$ & $10(16.7)$ & 0.19 \\
\hline Vancomycin & $1(1.7)$ & $1(1.7)$ & 0.98 \\
\hline Contrast & $8(13.8)$ & $11(18.3)$ & 0.50 \\
\hline Aminoglycosides & $2(3.4)$ & $2(3.3)$ & 0.97 \\
\hline Amphotericin & $2(3.4)$ & $0(0)$ & 0.15 \\
\hline NSAIDs & $2(3.4)$ & $1(1.7)$ & 0.54 \\
\hline Cardiac surgery, $n(\%)$ & $13(22.4)$ & $8(13.3)$ & 0.20 \\
\hline Treatment limitation, $n(\%)^{\mathrm{b}}$ & $12(20.7)$ & $10(16.7)$ & 0.58 \\
\hline Fluid accumulation at randomization $(\mathrm{mL})$, median (IQR) & $4763(2837-8515)$ & $5114(2050-8803)$ & 0.84 \\
\hline Percentage of fluid overload, median (IQR) ${ }^{c}$ & $9.53(3.43-19.68)$ & $7.63(2.10-12.02)$ & 0.87 \\
\hline Baseline NGAL (ng/mL), median (IQR) & $625(376-1362)$ & $860(447-1204)$ & 0.63 \\
\hline Baseline NT-proBNP (pg/mL), median (IQR) & $4301(515-35,000)$ & $5844(869-10,007)$ & 0.71 \\
\hline Baseline angiopoietin-2 (ng/mL), median (IQR) & $16,784(8649-35,545)$ & $22,294(12539-33,186)$ & 0.95 \\
\hline
\end{tabular}

Significant values are shown in bold type

AKI, acute kidney injury; APACHE II, Acute Physiology and Chronic Health Evaluation; GFR, glomerular filtration rate; ICU, intensive care unit; IQR, interquartile range; NGAL, neutrophil gelatinase-associated lipocalin; NSAID, nonsteroidal anti-inflammatory drug; NT-proBNP, N-terminal prohormone of brain natriuretic

peptide; RRT, renal replacement therapy; SD, standard deviation; SOFA, Sequential Organ Failure Assessment

aEstimated GFR was calculated by the CKD-EPI creatinine equation (2009)

'Treatment limitation is defined as withholding or withdrawal of patients from the treatment of primary disease either by the surrogates' decision or after a period of intensive care management

${ }^{c}$ Fluid overload is calculated by the total volume of fluid accumulation (intake - output) since ICU admission divided by body weight on admission and reported as a percentage 
Table 3 Duration parameters in the intervention trial

\begin{tabular}{llll}
\hline Parameters & Early RRT $(n=58)$ & Standard RRT $(n=60)$ & $P$ value \\
\hline Time from randomization to RRT (h), median (IQR) & $2(1-3)$ & $21(16.75-48.5)$ & $100(25-257)$ \\
Time from ICU admission to RRT (h), median (IQR) & $22(14-51)$ & $17(11-24)$ & $37.5(30-55)$ \\
Time from oliguria to RRT (h), median (IQR) & $4763(2837-8515)$ & $8659(4388-10,465)$ & $<0.001$ \\
Fluid accumulation from randomization to RRT (mL), median (IQR) & 0.001 \\
\hline
\end{tabular}

$\mathrm{ICU}$, intensive care unit; IQR, interquartile range; RRT, renal replacement therapy

sample may have been too small to detect some adverse events. For example, there was more hemodynamic instability (34.5\% versus $20 \%$ ) with early initiation while less cumulative fluid removal was seen at 7 days with standard RRT (1.2 versus $1.7 \mathrm{~L}$ ), but neither of these differences were significant. However, there was a significant difference in fluid accumulation from randomization to initiation of RRT ( 4.8 versus $8.7 \mathrm{~L}, P=0.02$ ) favoring early initiation.

Our final sample size was insufficient to test whether timing of initiation of RRT impacted 28-day survival. However, we did not observe any differences in survival between early and standard RRT (Table 4, Fig. 2). Other secondary outcomes including renal recovery rate, ICUfree days, mechanical ventilator-free days, 7-day fluid balance, and dialysis dependence rate were not significantly different between both treatment arms (Table 4), although we were unpowered for many of these endpoints. For example, ventilator-free days and ICU-free days were both greater with early initiation but with very wide confidence intervals. Similarly, the small differences observed in ICU and hospital length of stay (difference of 1.5 and 2.5 days, respectively) are clinical relevant but would have required a much larger trial to detect.

The optimal timing to initiate RRT in AKI patients remains to be established $[7,8,16-25]$. Two recently

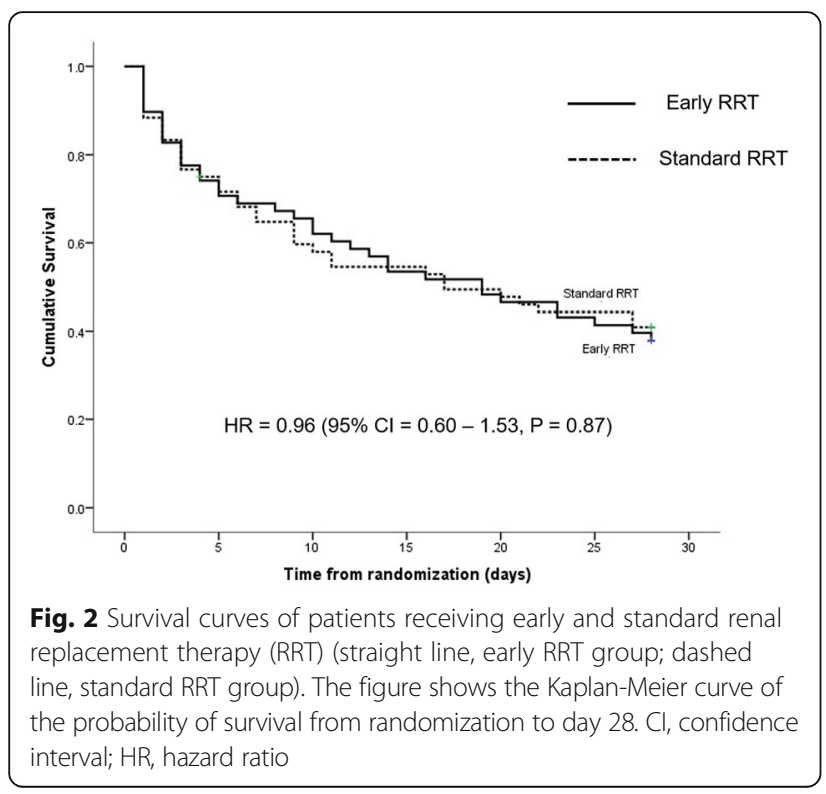

published RCTs examining timing of RRT initiation reached different conclusions. The AKIKI multicenter trial in France investigated early initiation (within $6 \mathrm{~h}$ after documentation of KDIGO stage 3) versus a "wait and see" strategy (as per conventional indications). Sepsis, severe sepsis, or septic shock were present in $80 \%$. Mortality at 60 days was not different between the two strategies [8]. Conversely, a single center RCT in Germany (ELAIN study) defined early RRT as AKI KDIGO stage 2 plus plasma NGAL $>150 \mathrm{ng} / \mathrm{mL}$ and delayed RRT as AKI stage 3. Early initiation of RRT significantly reduced 90-day mortality compared with delayed initiation (39.3\% versus 54.7\%) [7]. The other ongoing study, Standard versus Accelerated initiation of Renal Replacement Therapy in Acute Kidney Injury (STARRT-AKI), also uses the higher cut-off level of plasma NGAL ( $\geq 400 \mathrm{ng} / \mathrm{mL}$ ) as one of the three inclusion criteria along with a twofold rise in serum creatinine and oliguria [26]. By using plasma NGAL as a screening biomarker to filter patients, the ELAIN trial was able to select $90 \%$ of patients in the standard arm who required RRT. On the contrary, $49 \%$ of the patients in the standard indication arm of the AKIKI trial, which used only AKI staging as a screening tool, showed spontaneous recovery, which implied that RRT could also be avoided in some patients in the early indication arm had there been screening tools for selection of high-risk patients. Therefore, a pure clinical strategy may not be enough to analyze early versus standard initiation strategy and prevent unnecessary RRT. In our study, we identified 44/162 (27.2\%) FST-responsive patients with only a $13.6 \%$ rate of RRT. RRT was averted in $86.4 \%$ of FST responders. We were able to select a group with a 75\% RRT rate in the standard RRT group of FST nonresponders. In the standard arm, plasma NGAL was also a significant predictor for spontaneous recovery. This suggests that while FST is an excellent strategy to select patients who would recover, combining FST nonresponsiveness with plasma NGAL might be an even more suitable strategy to predict patients who are likely to require RRT.

There are some limitations in our study. First, due to the nature of the study, this was an unblinded RCT. The robust protocol for initiation of RRT and high compliance rates minimizes the risk of bias in RRT initiation. Second, the numbers of participants were rather small (60 in each arm) leading to insufficient power for secondary 
Table 4 Outcomes in the intervention trial

\begin{tabular}{|c|c|c|c|}
\hline Outcomes & Early RRT $(n=58)$ & Standard RRT $(n=60)$ & $p$ value \\
\hline \multicolumn{4}{|l|}{ Primary outcome } \\
\hline Mortality, n (\%) & $36(62.1)$ & $35(58.3)$ & 0.68 \\
\hline \multicolumn{4}{|l|}{ Secondary outcomes } \\
\hline Recovery, $n(\%)$ & $21(36.2)$ & $19(31.7)$ & 0.60 \\
\hline 7-day fluid balance (mL), median (IQR) & $-1702(-5610$ to 2129$)$ & $-1247(-4535$ to 1581$)$ & 0.75 \\
\hline Mean RRT dose (mL/kg/h), mean (SD) & $26.8(5.3)$ & $26.3(8.9)$ & 0.73 \\
\hline RRT-free days, median (IQR) & $0(0-19)$ & $0(0-28)$ & 0.64 \\
\hline MV-free days, median (IQR) & $4(0-24)$ & $0.5(0-20.3)$ & 0.66 \\
\hline ICU-free days, median (IQR) & $14(0-21)$ & $4.5(0-18)$ & 0.46 \\
\hline ICU length of stay (days), median (IQR) & $12(7-26)$ & $13.5(9-29)$ & 0.76 \\
\hline Hospital length of stay (days), median (IQR) & $26(19-53)$ & $28.5(17-55.3)$ & 0.82 \\
\hline Renal replacement therapy dependency at day $28, n(\%)$ & $7(12.1)$ & $10(16.7)$ & 0.77 \\
\hline
\end{tabular}

$\mathrm{ICU}$, intensive care unit; IQR, interquartile range; $\mathrm{MV}$, mechanical ventilation; RRT, renal replacement therapy

endpoints. However, as a pilot study, our results support the feasibility and safety of this approach for a definitive trial in the future. We were likely underpowered for our exploratory biochemical analysis as well. The incidence of hypophosphatemia was higher in the early RRT group, and severe hypophosphatemia is known to be associated with respiratory failure and weaning failure [27]. Plasma NGAL, NT-proBNP, and angiopoietin-2 levels in the early intervention arm were not significantly different from standard RRT. However, there were wide confidence intervals and important differences could have been missed.

\section{Conclusion}

This is the first pilot study to demonstrate the use of FST to identify patients with high risk of AKI progression and to investigate whether early RRT could improve clinical outcomes in this subgroup of patients. FST had excellent predictive ability for the subsequent use of RRT. Larger trials powered for clinical outcomes should be enabled by our results.

\section{Additional file}

Additional file 1: Table S1. Demographic, clinical, and biochemical data between FST-nonresponsive and FST-responsive patients. Table S2. Multivariable logistic regression on parameters to predict RRT. Table S3. Comparison of severity score and plasma biomarkers in the intervention trial. Table S4. Adverse events in the intervention trial. Appendix $\mathbf{1 .}$ Inclusion and exclusion criteria for patients in the FST study. Appendix 2. Definitions of safety outcomes related to the administration of RRT or vascular access for RRT. Figure S1. Survival curves of patients in the standard RRT arm who received and did not receive RRT (blue line, no RRT group; red line, RRT group). The figure shows the Kaplan-Meier curve of the probability of survival from randomization to day 28. (DOCX $520 \mathrm{~kb}$ )

\section{Abbreviations}

AKI: Acute kidney injury; AKIKI: Artificial Kidney Initiation in Kidney Injury; APACHE II: Acute Physiology and Chronic Health Evaluation; AUC: Area under the curve; Cl: Confidence interval; CVC: Central venous catheter;
CWH: Continuous venovenous hemofiltration; ELAIN: Effect of Early vs Delayed Initiation of Renal Replacement Therapy on Mortality in Critically III Patients with Acute Kidney Injury; FST: Furosemide stress test; HR: Hazard ratio; ICU: Intensive care unit; IQR: Interquartile range; KDIGO: Kidney Disease Improving Global Outcomes; NGAL: Neutrophil gelatinase-associated lipocalin; NT-proBNP: N-terminal prohormone of brain natriuretic peptide; PIRRT: Prolonged intermittent renal replacement therapy; RCT: Randomized controlled trial; RRT: Renal replacement therapy; SD: Standard deviation; SOFA: Sequential Organ Failure Assessment

\section{Acknowledgements}

We thank the staff, fellows, nurses, and research coordinators at Chulalongkorn University, Vajira Hospital, Nakhon Ping Hospital, Vajira Phuket Hospital, Bhumibol Adulyadej Hospital. We also thank Miss Sasipha Tachaboon, M.Sc., the medical technologist and Miss Pimnara Peerawaranun for statistical analysis.

\section{Funding}

This study is a part of a project supported by the Excellence Center for Critical Care Nephrology, King Chulalongkorn Memorial Hospital, and is funded by the National Kidney Foundation of Thailand.

\section{Availability of data and materials}

All data generated and/or analyzed during this study are included in this published article.

\section{Authors' contributions}

$\mathrm{NL}, \mathrm{KT}, \mathrm{KP}, \mathrm{KT}$, and NS designed the study; NL, SP, TT, WP, KS, AC, and PT carried out experiments; NL made the figures; NL, SE, JAK, and NS drafted and revised the paper; all authors read and approved the final manuscript.

Ethics approval and consent to participate

The study was approved by the Institutional Review Board of every participating center.

\section{Consent for publication}

The manuscript has been read and its submission approved by all co-authors. Patients were prospectively included with informed consent.

\section{Competing interests}

JAK reports grant support and consulting fees from Baxter and NxStage, unrelated to this study. The remaining authors declare that they have no competing interests.

\section{Publisher's Note}

Springer Nature remains neutral with regard to jurisdictional claims in published maps and institutional affiliations. 


\section{Author details}

'Division of Nephrology, Department of Medicine, Faculty of Medicine, Chulalongkorn University, Bangkok, Thailand. ${ }^{2}$ Excellence center for Critical Care Nephrology, King Chulalongkorn Memorial Hospital, Thai Red Cross Society, Bangkok, Thailand. ${ }^{3}$ Renal Division, Department of Medicine, Vajira Hospital, Navamindradhiraj University, Bangkok, Thailand. ${ }^{4}$ Nakhon Ping Hospital, Chiang Mai, Thailand. ${ }^{5}$ Bhumibol Adulyadej Hospital, Bangkok, Thailand. ${ }^{6}$ Vajira Phuket Hospital, Phuket, Thailand. ${ }^{7}$ The Center for Critical Care Nephrology, CRISMA, Department of Critical Care Medicine, University of Pittsburgh School of Medicine, Pittsburgh, PA, USA.

Received: 12 February 2018 Accepted: 27 March 2018 Published online: 19 April 2018

\section{References}

1. Hoste EA, Clermont G, Kersten A, Venkataraman R, Angus DC, De Bacquer D Kellum JA. RIFLE criteria for acute kidney injury are associated with hospital mortality in critically ill patients: a cohort analysis. Crit Care. 2006;10(3):R73.

2. Bagshaw SM, Laupland KB, Doig CJ, Mortis G, Fick GH, Mucenski M, Godinez-Luna T, Svenson LW, Rosenal T. Prognosis for long-term survival and renal recovery in critically ill patients with severe acute renal failure: a population-based study. Crit Care. 2005;9(6):R700-9.

3. Uchino S, Kellum JA, Bellomo R, Doig GS, Morimatsu H, Morgera S, Schetz M, Tan I, Bouman C, Macedo E, et al. Acute renal failure in critically ill patients: a multinational, multicenter study. JAMA. 2005;294(7):813-8.

4. Bagshaw SM, Wald R. Strategies for the optimal timing to start renal replacement therapy in critically ill patients with acute kidney injury. Kidney Int. 2017;91(5):1022-32

5. Shiao CC, Huang TM, Spapen HD, Honore PM, Wu VC. Optimal timing of renal replacement therapy initiation in acute kidney injury: the elephant felt by the blindmen? Crit Care. 2017;21(1):146.

6. Macedo E, Mehta RL. Timing of dialysis initiation in acute kidney injury and acute-on-chronic renal failure. Semin Dial. 2013;26(6):675-81.

7. Zarbock A, Kellum JA, Schmidt C, Van Aken H, Wempe C, Pavenstadt H, Boanta A, Gerss J, Meersch M. Effect of early vs delayed initiation of renal replacement therapy on mortality in critically ill patients with acute kidney injury: the ELAIN randomized clinical trial. JAMA. 2016;315(20):2190-9.

8. Gaudry S, Hajage D, Schortgen F, Martin-Lefevre L, Pons B, Boulet E, Boyer A, Chevrel G, Lerolle N, Carpentier D, et al. Initiation strategies for renal-replacement therapy in the intensive care unit. $N$ Engl J Med. 2016:375(2):122-33.

9. Chawla LS, Davison DL, Brasha-Mitchell E, Koyner JL, Arthur JM, Shaw AD, Tumlin JA, Trevino SA, Kimmel PL, Seneff MG. Development and standardization of a furosemide stress test to predict the severity of acute kidney injury. Crit Care. 2013;17(5):R207.

10. Koyner $\mathrm{L}$, Davison DL, Brasha-Mitchell E, Chalikonda DM, Arthur JM, Shaw AD, Tumlin JA, Trevino SA, Bennett MR, Kimmel PL, et al. Furosemide stress test and biomarkers for the prediction of AKI severity. J Am Soc Nephrol. 2015;26(8):2023-31.

11. Group KDIGOKAKIW. KDIGO clinical practice guideline for acute kidney injury. Kidney Int. 2012;2(1):6.

12. Network TVNARFT. Intensity of renal support in critically ill patients with acute kidney injury. N Engl J Med. 2008;359(1):7-20.

13. Chen H, Wu B, Gong D, Liu Z. Fluid overload at start of continuous renal replacement therapy is associated with poorer clinical condition and outcome: a prospective observational study on the combined use of bioimpedance vector analysis and serum $\mathrm{N}$-terminal pro-B-type natriuretic peptide measurement. Crit Care. 2015;19:135.

14. Cruz DN, de Cal M, Garzotto F, Perazella MA, Lentini P, Corradi V, Piccinni P, Ronco C. Plasma neutrophil gelatinase-associated lipocalin is an early biomarker for acute kidney injury in an adult ICU population. Intensive Care Med. 2010;36(3):444-51.

15. Kumpers P, Hafer C, David S, Hecker H, Lukasz A, Fliser D, Haller H, Kielstein JT, Faulhaber-Walter R. Angiopoietin-2 in patients requiring renal replacement therapy in the ICU: relation to acute kidney injury, multiple organ dysfunction syndrome and outcome. Intensive Care Med. 2010;36(3):462-70.

16. Besen BAMP, Romano TG, Mendes PV, Gallo CA, Zampieri FG, Nassar AP Jr, Park M. Early Versus Late Initiation of Renal Replacement Therapy in Critically III Patients: Systematic Review and Meta-Analysis. J Intensive Care Med. 2017. https://doi.org/10.1177/0885066617710914. [Epub ahead of print].
17. Bhatt GC, Das RR. Early versus late initiation of renal replacement therapy in patients with acute kidney injury - a systematic review and meta-analysis of randomized controlled trials. BMC Nephrol. 2017;18(1):78.

18. Boussekey N, Capron B, Delannoy PY, Devos P, Alfandari S, Chiche A, Meybeck A, Georges H, Leroy O. Survival in critically ill patients with acute kidney injury treated with early hemodiafiltration. Int J Artif Organs. 2012;35(12):1039-46.

19. Feng YM, Yang Y, Han XL, Zhang F, Wan D, Guo R. The effect of early versus late initiation of renal replacement therapy in patients with acute kidney injury: a meta-analysis with trial sequential analysis of randomized controlled trials. PLoS One. 2017;12(3):e0174158.

20. Karvellas CJ, Farhat MR, Sajjad I, Mogensen SS, Leung AA, Wald R, Bagshaw SM. A comparison of early versus late initiation of renal replacement therapy in critically ill patients with acute kidney injury: a systematic review and meta-analysis. Crit Care. 2011;15(1):R72.

21. Leite TT, Macedo E, Pereira SM, Bandeira SR, Pontes PH, Garcia AS, Militao FR, Sobrinho IM, Assuncao LM, Liborio AB. Timing of renal replacement therapy initiation by AKIN classification system. Crit Care. 2013;17(2):R62.

22. Shiao CC, Wu VC, Li WY, Lin YF, Hu FC, Young GH, Kuo CC, Kao TW, Huang DM, Chen YM, et al. Late initiation of renal replacement therapy is associated with worse outcomes in acute kidney injury after major abdominal surgery. Crit Care. 2009;13(5):R171.

23. Shum HP, Chan KC, Kwan MC, Yeung AW, Cheung EW, Yan WW. Timing for initiation of continuous renal replacement therapy in patients with septic shock and acute kidney injury. Ther Apher Dial. 2013;17(3):305-10.

24. Wald R, Adhikari NKJ, Smith OM. Comparison of standard and accelerated initiation of renal replacement therapy in acute kidney injury. Kidney Int. 2015;88(4):897-904.

25. Wu SC, Fu CY, Lin HH, Chen RJ, Hsieh CH, Wang YC, Yeh CC, Huang HC, Huang JC, Chang YJ. Late initiation of continuous veno-venous hemofiltration therapy is associated with a lower survival rate in surgical critically ill patients with postoperative acute kidney injury. Am Surg. 2012;78(2):235-42.

26. Smith OM, Wald R, Adhikari NK, Pope K, Weir MA, Bagshaw SM. Standard versus accelerated initiation of renal replacement therapy in acute kidney injury (STARRT-AKI): study protocol for a randomized controlled trial. Trials. 2013;14:320.

27. Alsumrain MH, Jawad SA, Imran NB, Riar S, DeBari VA, Adelman M. Association of hypophosphatemia with failure-to-wean from mechanical ventilation. Ann Clin Lab Sci. 2010;40(2):144-8.

\section{Submit your next manuscript to BioMed Central and we will help you at every step:}

- We accept pre-submission inquiries

- Our selector tool helps you to find the most relevant journal

- We provide round the clock customer support

- Convenient online submission

- Thorough peer review

- Inclusion in PubMed and all major indexing services

- Maximum visibility for your research

Submit your manuscript at www.biomedcentral.com/submit
) Biomed Central 